\title{
Interpreting magnetization from Faraday rotation in birefringent, magnetic media
}

\author{
S. R. Woodford,* A. Bringer, and S. Blügel \\ Institut für Festkörperforschung, Forschungszentrum Jülich, D-52425 Jülich, Germany
}

(Dated: June 8, 2006)

\begin{abstract}
The Faraday effect is an extremely useful probe of magnetization dynamics on an ultrafast scale. However, in birefringent materials, interpreting experimental results is nontrivial. We investigate the link between magnetization and Faraday rotation by solving Maxwell's equations in a magneticallyordered, birefringent material. We find that the periodic dependence of the Faraday rotation on the sample thickness (a well-known effect of birefringence) complicates the correspondence between the sample magnetization and the measured rotation; in particular, the normalization constant for comparing magnetization and rotation depends on the sample thickness. Furthermore, sample alignment becomes important. If the incident light is not polarized along a birefringence axis of the sample, then the magnetization can be correctly interpreted only if the dielectric tensor is very accurately known.
\end{abstract}

PACS numbers: $78.20 \mathrm{Ls}, 78.20 \mathrm{Fm}$

\section{INTRODUCTION}

In the search to increase the speed of the read- and write-processes in magnetic data storage, a number of different methods have been proposed. Precessional switching, which makes use of a short, intense magnetic pulse, has been demonstrated to reliably switch the magnetization direction on a time scale of around 200 ps [1-3]. Unfortunately, generation of strong magnetic pulses shorter than around $100 \mathrm{ps}$ is out of the range of modern experiments [4], which determines the maximum speed at which switching may be achieved. On the other hand, extremely short laser pulses can be generated (with a FWHM of lower than $50 \mathrm{fs}$ ) and demagnetization arising from the absorption of such a pulse appears to occur within 300 fs (see e.g. [5-7]) after the absorption. However, absorption of a laser pulse causes heating, and any magnetic storage device that is switched in this manner will suffer a long down-time between write cycles, to allow the system to cool.

Recently, excitation of magnetic oscillations through nonthermal optical processes has been demonstrated in $\mathrm{DyFeO}_{3}$ [8], a canted antiferromagnet, with $M_{s} \approx$ $8 \mathrm{G}$. This indicates the possibility of obtaining magnetic switching on the time-scale of a laser pulse, while not requiring time for heat dissipation. The excitation process makes use of the inverse Faraday effect: circularly polarised light propagating through a (nonresonant) medium undergoes Raman scattering via spin-orbit split excited states, generating an effective magnetic field. The strength of this field depends on the magnetooptical response of the medium, which is also responsible for the Faraday effect (where the polarization axis of plane polarized light is rotated according to the material's magnetization) - this explains the name "inverse Faraday effect".

\footnotetext{
*Electronic address: s.woodford@fz-juelich.de
}

Phenomenologically, the connection between the Faraday effect and inverse Faraday effect is well understood (see e.g. $[9,10]$ ). Using the phenomenological equations, Kimel et al calculate the strength of their effective magnetic field to be around $0.3 \mathrm{~T}$, with a FWHM of 200 fs [8]. They measure the magnetization dynamics using Faraday rotation, and they estimate the amplitude of magnetic oscillations as $\left(M-M_{s}\right) / M_{s} \approx 1 / 16$. The effect of their "effective magnetic pulse" can also be simulated using a Landau-Lifshitz equation [11], which yields a similar amplitude.

Despite this consistency, there are certain aspects of the interpretation of Faraday rotation measurements that pass unmentioned in [8], which we believe deserve more attention. This is particularly true for the orthoferrites, which are not only birefringent $[12,13]$ but also have enhanced magnetooptical properties due to the so-called "anisotropic orbital quenching" [14]. To obtain a reasonable estimate of the amplitude of magnetization dynamics, these effects must be taken into account.

It is well known that birefringence limits the rotation angle of plane-polarized light (see e.g. [15]); instead of being linearly proportional to the sample thickness $d$, the rotation is a periodic function of $d$, and can vanish if $d$ is poorly chosen. Anisotropic orbital quenching is a proposed explanation for the antiferromagnetic Faraday effect, where the antiferromagnetic vector $\mathbf{l}$ causes rotation of the light's polarization plane. (Symmetry considerations show that this effect is only possible in materials with inequivalent magnetic sublattices, like the orthoferrites [16].) This rotation opposes the rotation caused by the magnetic vector $\mathbf{m}$, and leads to a reduced observable rotation [17].

For clarity of presentation, and to allow our results to be directly applicable to the experiment described in [8], we will assume our sample has the magnetic- and crystal structure of the orthoferrites. More precisely, we consider an orthorhombic crystal, antiferromagnetically ordered with the antiferromagnetic vector $\mathbf{l}$ directed along the $a$-axis. Due to a small canting of the antiferromag- 
netic sublattices, there is a weak residual ferromagnetic component $\mathbf{m}$ directed along the $c$-axis (for more details, see [18]). Birefringence is due to the crystal structure, and the birefringence axes coincide with the crystal axes. We will consider a light beam travelling parallel to the $c$-axis. We are interested in near-equilibrium properties, so that the dynamics of the antiferromagnetic vector will have a negligible effect. Furthermore, we will assume that the ferromagnetic vector remains directed along $c$ at all times, so that only its magnitude may vary. Therefore $\mathbf{l}=l \hat{\mathbf{a}}$ and $\mathbf{m}=m \hat{\mathbf{c}}$, with $l$ a constant and $m$ a variable. In what follows, we use the convention that $x, y$, and $z$ represent the $a, b$ and $c$ directions of the orthorhombic structure, respectively.

\section{FARADAY ROTATION IN A BIREFRINGENT WEAK FERROMAGNET}

It is well known $[10,15,19]$ that after propagating through an isotropic, non-magnetically ordered material, plane polarized light remains plane-polarized, and has its polarization plane rotated by the angle

$$
\beta=V H d
$$

where $d$ is the path length of the light, $H$ is an external magnetic field, and $V$ is the Verdet constant, which describes magnetooptical activity. This rotation is referred to as the "Faraday effect". Closely related to this is the "inverse Faraday effect" (these effects can be related by thermodynamic arguments [9]), where circularly polarized light passing through a medium generates a magnetization according to the relation

$$
M=\lambda_{0} V(2 \pi c)^{-1}\left(I_{R}-I_{L}\right) .
$$

Here, $\lambda_{0}$ is the vacuum wavelength of the light, and $I_{R, L}$ are the intensities of right- and left-circularly polarized light respectively. We note that both Eq.(1) and Eq.(2) are only valid when the material is isotropic, optically inactive and non-magnetically ordered. We now explore how Eq.(1) changes as the birefringence and magnetic order are introduced. (Since we are most interested in the orthoferrites, which are optically inactive, we do not investigate the effect of optical activity.)

Orthoferrites are orthorhombic structures. In the absence of magnetic order-, the dielectric tensor is symmetric and diagonal: $\epsilon=\operatorname{diag}\left(\epsilon_{x}, \epsilon_{y}, \epsilon_{z}\right)$. In general, $\epsilon_{x} \neq \epsilon_{y}$, and the material is birefringent. (We consider light propagating along the $z$-axis, and so $\epsilon_{z}$ does not enter our analysis.) Magnetic ordering introduces antisymmetric (and therefore off-diagonal) terms into this tensor [16]. It can be shown (see e.g. [20, 21] and the references therein) that the antisymmetric part of $\epsilon_{i j}$ is (proportional to) the dual of the ferromagnetic vector $\mathbf{m}$ (i.e. $\epsilon_{x y}$ contains $m_{z}$, etc.). This is not true for the antiferromagnetic vector 1 - since $l_{x}$ transforms identically to $m_{z}$, it follows $[20,21]$ that $l_{x}$ enters $\epsilon_{x y}$ (and not $\epsilon_{y z}$, as we would naively expect).
As noted above, we assume that the deviation from magnetic equilibrium is small. In this case, the antiferromagnetic vector $\mathbf{l}$ is well described by a vector of fixed length and direction. On the other hand, the ferromagnetic vector $\mathbf{m}$, which is the result of a small canting of the antiferromagnetic order, has variable length and direction. To simplify the exposition, we fix the direction of $\mathbf{m}$ and allow only the magnitude to vary, i.e. $\mathbf{m}=(0,0, m)$. Under these assumptions, $\epsilon_{x z}=\epsilon_{y z}=0$, and

$$
\epsilon_{x y}=-\epsilon_{y x}=i\left(f_{1}+\xi_{1} \frac{m}{m_{0}}\right)
$$

The phenomenological parameters $f_{1}$ and $\xi_{1}$ describe the antiferromagnetic and ferromagnetic contributions to $\epsilon_{x y}$ respectively, and $m_{0}$ is the equilibrium magnitude of $\mathbf{m}$. We introduce the "ferromagnetic deviation" $\kappa=(m-$ $\left.m_{0}\right) / m_{0}$, and write Eq.(3) as

$$
\epsilon_{x y}=i \gamma_{0}(1+\zeta \kappa) \equiv i \gamma
$$

where $\gamma_{0}=\left(f_{1}+\xi_{1}\right)$ describes the equilibrium magnetooptical properties of the system, and $\zeta=\xi_{1} / \gamma_{0}$ measures the ferromagnetic contribution to magneto-optics. Experimental values of $\gamma_{0}$ for the various rare-earth orthoferrites at different wavelengths of light can be found in Ref.[13]. The parameter $\zeta$ is more difficult to find in the literature; it has been evaluated as $\zeta \approx 0.23 \pm 0.13$ for $\mathrm{YFeO}_{3}$ at $T=295 \mathrm{~K}$, using light with wavelength $\lambda=0.6328 \mu \mathrm{m}[21]$. This value should vary with frequency and temperature, although it should not vary significantly for different rare-earths, as these have negligible effect on the magnetic order at high temperatures [22]. Since the dependence of $\zeta$ on temperature and frequency is not known, we fix $\zeta=0.23$ for the remainder of this paper.

We note that Eq.(4) is valid provided the magnetic field that caused the change of magnetization from equilibrium has stopped acting, i.e. Eq.(4) holds while the system relaxes towards equilibrium. If the magnetic field is still present, then a diamagnetic term should be added [21]. This was neglected in Ref.[17], which explains why their estimate $\zeta \approx 0.13$ differs from that found in Ref.[21].

With the above assumptions in place, we may write $[12,15]$

$$
\mathbf{D}=\left(\begin{array}{ccc}
\epsilon+\eta & i \gamma & 0 \\
-i \gamma & \epsilon-\eta & 0 \\
0 & 0 & \epsilon_{z}
\end{array}\right) \mathbf{E}
$$

the strength of the birefringence is determined by $\eta$. Assuming no conduction current exists, $\mathbf{D}$ and $\mathbf{E}$ obey the wave equation

$$
\mu \frac{\partial^{2} \mathbf{D}}{\partial t^{2}}=\nabla^{2} \mathbf{E} .
$$

(All magnetooptical effects have been placed in the dielectric tensor, and therefore the permeability $\mu$ is a 
scalar quantity [23].) We solve Eq.(6) for a plane wave propagating along the $z$-axis:

$$
\mathbf{E}=\left(\begin{array}{c}
E_{x} \\
E_{y} \\
0
\end{array}\right) e^{i(\omega t-k z)}
$$

Substituting (7) into Eq.(6) and using Eq.(5), we obtain

$$
\left(\begin{array}{cc}
\epsilon+\eta-k^{2} / \omega^{2} \mu & i \gamma \\
-i \gamma & \epsilon-\eta-k^{2} / \omega^{2} \mu
\end{array}\right)\left(\begin{array}{c}
E_{x} \\
E_{y}
\end{array}\right)=0
$$

This equation provides us with both the admissible wavevectors

$$
k_{ \pm}=\sqrt{\omega^{2} \mu\left(\epsilon \pm \sqrt{\eta^{2}+\gamma^{2}}\right)}
$$

and the normal modes in the material:

$$
\begin{aligned}
& \mathbf{n}_{+} \equiv\left(\begin{array}{c}
E_{x} \\
E_{y}
\end{array}\right)_{+}=\left(\begin{array}{c}
1 \\
i\left(\Gamma-\sqrt{1+\Gamma^{2}}\right)
\end{array}\right) e^{i\left(\omega t-k_{+} z\right)} \\
& \mathbf{n}_{-} \equiv\left(\begin{array}{c}
E_{x} \\
E_{y}
\end{array}\right)_{-}=\left(\begin{array}{c}
1 \\
i\left(\Gamma+\sqrt{1+\Gamma^{2}}\right)
\end{array}\right) e^{i\left(\omega t-k_{-} z\right)}
\end{aligned}
$$

Here we have introduced $\Gamma=\eta / \gamma$ to describe the relative strength of the birefringence compared with magnetic effects. The medium admits two normal modes, which are elliptically polarized and which propagate at different velocity ( since $k_{+} \neq k_{-}$). The difference in velocities is related to the magnitudes of $\gamma$ and $\eta$, and therefore to both the magnetization and the birefringence. Note that since $\epsilon_{x z}=0=\epsilon_{y z}$, the $z$-component of the light always vanishes. In general, the light will emerge from the material elliptically polarized; "rotation" of the light is therefore defined by the change of direction of the major axis of the polarization ellipse.

Now, assume that plane polarized light is normally incident at $z=0$, i.e.

$$
\mathbf{E}_{z=0}=|E|\left(\begin{array}{c}
\cos \theta \\
\sin \theta
\end{array}\right) e^{i \omega t},
$$

where $\theta$ is the angle between the polarization plane and the $x$-axis. Upon entering the material, this decomposes into normal modes

$$
\mathbf{E}_{z=0}=\frac{1}{2}|E|\left(\alpha_{+} \mathbf{n}_{+, z=0}+\alpha_{-} \mathbf{n}_{-, z=0}\right) ;
$$

$\alpha_{ \pm}$are complex parameters, which are easily determined using eq.(10). Furthermore, these equations also describe the propagation of the wave through the medium. Since we are interested in determining the rotation of the light relative to its initial angle, it is convenient to rotate to the coordinate frame $\left(x^{\prime}, y^{\prime}\right)$ in which the light is initially polarized along the $x^{\prime}$-axis. In this new coordinate frame, after the light has propagated a distance $d$, the electric field vector is

$$
\mathbf{E}_{z=d}=\frac{|E| e^{i(\omega t-\bar{k} d)}}{\sqrt{1+\Gamma^{2}}}\left(\begin{array}{c}
\sqrt{1+\Gamma^{2}} \cos \delta d-i \Gamma \sin \delta d \cos 2 \theta \\
-\sin \delta d+i \Gamma \sin \delta d \sin 2 \theta
\end{array}\right)
$$

where $\bar{k}=\left(k_{+}+k_{-}\right) / 2$ and $\delta=\left(k_{+}-k_{-}\right) / 2$. Note that under the assumption that $\sqrt{\eta^{2}+\gamma^{2}} \ll \epsilon$ (which is true in most materials, including the orthoferrites), Eq.(9) allows us to rewrite the wave-vector mismatch $\delta$ as

$$
\delta \approx \frac{1}{2} \omega \sqrt{\frac{\mu}{\epsilon}} \sqrt{\gamma^{2}+\eta^{2}}
$$

The polarization ellipse of the outgoing light (12) is described by the lengths of the major and minor axes, and the orientation of the ellipse (see e.g. [24] for details). Relative to the initial angle (i.e. relative to the $x^{\prime}$-axis), the direction of polarization has rotated through the angle $\beta$, where

$$
\begin{array}{r}
\tan 2 \beta=\frac{2 \operatorname{Re}\left(E_{x}^{*} E_{y}\right)}{\left|E_{x}\right|^{2}-\left|E_{y}\right|^{2}} \\
=\frac{-\sqrt{1+\Gamma^{2}}[\sin 2 \delta d+\Gamma(1-\cos 2 \delta d) \sin 2 \theta \cos 2 \theta]}{\cos 2 \delta d\left[1+\Gamma^{2} \sin ^{2} 2 \theta\right]+\Gamma^{2} \cos ^{2} 2 \theta} .
\end{array}
$$

The lengths of the major axis $b_{+}$and minor axis $b_{-}$of the polarization ellipse are:

$$
\begin{aligned}
\frac{b_{ \pm}}{|E|}= & \frac{1}{|E|}\left(\sqrt{|E|^{2}+2 \operatorname{Im}\left(E_{x}^{*} E_{y}\right)} \pm \sqrt{|E|^{2}-2 \operatorname{Im}\left(E_{x}^{*} E_{y}\right)}\right) \\
& =\left\{1+\frac{\Gamma \sin 2 \delta d \sin 2 \theta}{\sqrt{1+\Gamma^{2}}}-\frac{\Gamma(1-\cos 2 \delta d) \cos 2 \theta}{1+\Gamma^{2}}\right\}^{1 / 2} \\
& \pm\left\{1-\frac{\Gamma \sin 2 \delta d \sin 2 \theta}{\sqrt{1+\Gamma^{2}}}+\frac{\Gamma(1-\cos 2 \delta d) \cos 2 \theta}{1+\Gamma^{2}}\right\}^{1 / 2}
\end{aligned}
$$

These equations are the key to investigating the effect of magnetization changes on the outgoing light beam. Since magnetic effects are described by $\gamma$, which is contained in both $\delta$ (see Eq.(13)) and $\Gamma=\eta / \gamma$, the dependence on magnetization is complicated, and analytical results are only available in certain limits.

\section{LIMITING CASES}

\section{A. Zero birefringence}

The first limit that should be examined is the case of zero birefringence. In this limit, $\Gamma=0$, and Eq.(14) reduces to

$$
\tan 2 \beta=-\tan 2 \delta d
$$

i.e. the polarization plane of the light rotates through an angle $\beta=-\delta d=-\frac{1}{2} d \omega \sqrt{\mu / \epsilon} \gamma$. Furthermore, the light emerges linearly polarized: $b_{-}=0$. We define the angle $\beta_{0}$ as the rotation caused when the sample is in magnetic equilibrium:

$$
\beta_{0}=-\frac{1}{2} d \omega \sqrt{\mu / \epsilon} \gamma_{0}
$$


From the definition of $\gamma(4)$, we have

$$
\beta=\beta_{0}+\beta_{0} \zeta \kappa
$$

the dependence of the angle $\beta$ on magnetization is contained in $\kappa=\left(m-m_{0}\right) / m_{0}$.

Eq.(18) is the analogue of Eq.(1) for magneticallyordered materials. The effect of magnetic ordering is most clear if we consider a magnetic configuration in which the magnetization vanishes. In the case of Eq.(1), this occurs for $H=0$, and there is no Faraday rotation. On the other hand, for Eq.(18) the magnetization vanishes at $\kappa=-1$, but Faraday rotation still occurs: $\beta=\beta_{0}(1-\zeta)$. The factor of $(1-\zeta)$ shows that this rotation is caused by the antiferromagnetic ordering of the inequivalent magnetic sublattices; $\zeta$ is the normalized ferromagnetic contribution to magneto-optics, so $(1-\zeta)$ is the antiferromagnetic contribution.

The Verdet constant, which describes the magnetooptical response of the material, is defined from the slope of $\beta(m)$. Introducing $m=\chi H$ in Eq.(1) (where $\chi$ is the magnetic susceptibility), we find that

$$
V=\frac{\chi}{d} \frac{\partial \beta}{\partial m} .
$$

From this definition, Eqs.(17)-(18) and letting $\chi$ now represent the $z z$-component of the susceptibility in the magnetic medium, we find

$$
V=-\frac{\chi \zeta \beta_{0}}{d m_{0}} .
$$

This parameter can be used in Eq.(2) to provide an estimate for the strength of the inverse Faraday effect.

Finally, we note that for the purposes of interpreting experimental data, it is more useful to rewrite Eq.(18) as

$$
\frac{\beta-\beta_{0}}{\beta_{0}}=\zeta \kappa=\zeta \frac{m-m_{0}}{m_{0}} .
$$

i.e. The change of rotation, normalized against the equilibrium rotation, is proportional to the change of magnetization, normalized to equilibrium ferromagnetic moment. The proportionality constant is $\zeta$, the ferromagnetic contribution to magneto-optics. Note that for zero birefringence, $\beta_{0}$ defined by Eq.(17) cannot vanish and Eq.(19) is always well-defined.

\section{B. Large birefringence, small $\theta$}

The second limit that we consider is the relevant limit for analysis of the orthoferrites, i.e. large birefringence. Indeed, the orthoferrites generally satisfy $\gamma_{0} \ll \eta$ (see e.g. [25]). For the particular setup described in [8], where $\mathrm{DyFeO}_{3}$ is probed by light with wavelength $\lambda_{0} \approx 0.7 \mu \mathrm{m}$, experimental results reveal that $\left|\gamma_{0} / \eta\right| \approx 1 / 30$ [13], so the parameter $\Gamma \approx 30$. It will be useful in the subsequent analysis to note that in equilibrium, this system has $\delta_{0}=$ $\frac{\pi}{20}$ rads $\mu \mathrm{m}^{-1}$ for light with wavelength $\lambda_{0}=0.7 \mu \mathrm{m}$, and $\zeta=0.23$, as mentioned above.

When birefringence is present, the polarization direction of the incident beam is important. Analysis of Eq.(14) for large $\Gamma$ and arbitrary $\theta$ is best performed numerically. In this section, we consider only the case where the angle $\theta$ is small, i.e. the incident light is polarized approximately parallel to the $x$-axis.

First, we quantify what we mean by "small $\theta$ ". Expanding Eq.(14) in $\kappa$, we find that the $\theta$ dependence can be neglected up to linear order in $\kappa$ provided

$$
\cot \delta_{0} d \gg \Gamma_{0} \sin 2 \theta
$$

where $\Gamma_{0}=\Gamma(\kappa=0)$ and $\delta_{0}=\delta(\kappa=0)$. Note the dependence of this condition on $d-$ for poorly chosen sample thickness, the experimental alignment must be extremely accurate.

To illustrate the order of magnitude of the critical value of $\theta$, we consider $\mathrm{DyFeO}_{3}$ with $d$ chosen so that $\cot \delta_{0} d \approx$ 1 for convenience. Then $\theta$-dependence can be neglected provided

$$
\sin 2 \theta \ll 1 / 30
$$

i.e. $\theta$ must be less than $1^{\circ}$ away from the birefringence axis. This accuracy in alignment is not difficult to achieve, and makes the subsequent analysis of experimental results far easier.

For $\theta$ small enough, Eq.(14) can be reduced to

$$
\beta=\beta_{0}(1-\zeta \kappa)-\zeta \kappa \cos 2 \delta_{0} d \frac{\delta_{0} d}{\left|\Gamma_{0}\right|^{3}}
$$

where

$$
\beta_{0}=-\sin 2 \delta_{0} d /\left|2 \Gamma_{0}\right|,
$$

and terms of $\mathcal{O}\left(\kappa^{2}\right)$ have been neglected. Eq.(22) shows the well known $([12,15])$ phenomenon that the rotation induced by the sample in equilibrium depends on the sample thickness periodically when birefringence is strong, not linearly as in the zero-birefringence case (compare Eq.(17)). We also note that the equilibrium $(\kappa=0)$ rotation is constrained to lie between two limiting angles $\left(\beta_{\max , \min }= \pm\left(2 \Gamma_{0}\right)^{-1}\right)$, another well known fact $[12]$.

Now consider the effect of magnetic deviations on the Faraday rotation angle. Since $\Gamma_{0}^{-3}$ is assumed to be small, we may neglect the final term in Eq.(21), except when $\beta_{0}$ is also small (i.e. for particular values of $d$ ). If the thickness of the sample is not near the value where $\beta_{0}$ vanishes, we have

$$
\frac{\beta-\beta_{0}}{\beta_{0}}=-\zeta \kappa
$$

which is identical to Eq.(19), up to a factor of -1 .

This change of sign arises because the magnetization enters Eq.(14) through two different parameters, the 
wave-vector mismatch $\delta$ and the strength of birefringence $\Gamma$ (relative to the strength of magnetic effects). Increasing the magnetization (i.e. increasing $\gamma$ ) will increase $\delta$ and decrease $\Gamma$. For zero birefringence, the rotation is governed by changes to $\delta$ (see Eq.(19)), while when birefringence is large, the rotation is dominated by changes in $\Gamma$. (This is clear from Eq.(21), where the first term in $\kappa$ arises from expanding $\Gamma$, while the second, smaller, $\kappa$-term is due to the change in $\delta$.) This explains the difference of sign between Eq.(19) and Eq.(23).

If Eq.(23) holds (i.e. if the light is accurately aligned and the equilibrium rotation $\beta_{0}$ is nonzero) then magnetization dynamics are easily inferred from the measured rotation. The accuracy of this correspondence is determined by the accuracy of measurement of $\beta$ and the accuracy with which $\zeta$, the ferromagnetic magneto-optical parameter, is known. It is important to note that the angle by which we normalize the rotation is not the limiting angle $\beta_{\max }$, but the equilibrium angle $\beta_{0}$, which depends both on the material and also on the sample thickness; $\beta_{0}$ must be measured for each different sample. Furthermore, we note that if $\beta_{0}$ is small (but still larger than $\Gamma_{0}^{-3}$, so that Eq.(23) holds), the change of rotation will be extremely small, even for large changes in the magnetization.

Eq.(23) is valid provided

$$
\sin 2 \delta_{0} d \gg \cos 2 \delta_{0} d \frac{2 \delta_{0} d}{\Gamma_{0}^{2}} .
$$

Since $\Gamma_{0}$ is large (birefringence is large), this condition fails when $\sin 2 \delta_{0} d$ is extremely small, i.e. $2 \delta_{0} d \approx n \pi$ (with $n$ an integer). Writing $2 \delta_{0} d=n \pi+\varphi,|\varphi| \ll 1$, we find that the Eq.(23) is invalid if

$$
|\varphi| \leq \frac{n \pi}{\Gamma_{0}^{2}}
$$

In this case, the final term in Eq.(21) must be included in the analysis. Depending on the sign of $\beta_{0}$, this additional term may either enhance or diminish the magnetizationdependent rotation. However, in either case it is clear from Eq.(21) that the rotation induced by the sample is extremely small, and variations in the rotation for changing magnetization will be difficult to measure reliably. The sample thickness should be chosen such that $\beta_{0}$ is large, and this problem is avoided.

We illustrate the above analysis using numerical values relevant to the experiment described in [8]. We define the parameter $P$ as the ratio between the change of rotation and the normalized change of magnetization, i.e.

$$
P \equiv \frac{\beta-\beta_{0}}{\kappa}=-\beta_{0} \zeta-\zeta \cos 2 \delta_{0} d \frac{\delta_{0} d}{\left|\Gamma_{0}\right|^{3}} .
$$

(See Eq.(21).) Table I shows the values of $\beta_{0}, P$ and $-\frac{\zeta \beta_{0}}{P}$ for several values of the sample thickness $d$. Note that if $P \approx-\zeta \beta_{0}$, then Eq.(23) is approximately valid.

From Table I, we see that small changes of the sample thickness may lead to large changes both in $\beta_{0}$ and
TABLE I: Theoretical values of $\beta_{0}$ and $P$ as a function of the thickness $d$ of a dysprosium orthoferrite sample, probed with light of wavelength $\lambda \approx 0.7 \mu \mathrm{m}$. We also provide values for $-\frac{\zeta \beta_{0}}{P}$; if this parameter is close to 1 , then Eq.(23) is approximately valid. For this material, $\beta_{\max } \approx 1^{\circ}$.

\begin{tabular}{cccc}
\hline \hline $\mathrm{d}(\mu \mathrm{m})$ & $\beta_{0}$ (degrees) & $\mathrm{P}($ degrees $)$ & $-\frac{\zeta \beta_{0}}{P}$ (dimensionless) \\
\hline 55 & 0.95 & -0.22 & 1.00 \\
58 & 0.56 & -0.13 & 0.97 \\
59 & 0.30 & -0.07 & 0.94 \\
60 & 0.00 & -0.004 & 0.00 \\
61 & -0.30 & 0.06 & 1.07 \\
62 & -0.56 & 0.13 & 1.03 \\
65 & -0.95 & 0.22 & 1.00 \\
\hline \hline
\end{tabular}

in $P$. However, $-\frac{\zeta \beta_{0}}{P}$ remains approximately constant for most values of $d$. Finally we note that $\beta_{0}$ and $P$ depend strongly on the values of $\Gamma_{0}$ and $\delta_{0}$, which we know only approximately. For this reason, our numerical results may be inaccurate; in particular, our estimates of the critical thicknesses for which $\beta_{0}$ vanishes are unlikely to be correct, and should rather be determined by experiment.

This sensitive dependence on the accuracy of $\delta_{0}$ and $\Gamma_{0}$ explains why it is preferable to work in a regime where Eq.(23) may be used, rather than trying to work directly with Eq.(14).

We conclude this section with a discussion of the frequency dependence of the above results. From Ref.[13], we find that the parameter $\delta_{0}$ (and, to a lesser extent, $\left.\Gamma_{0}\right)$ is frequency dependent. Thus if we measure a sample using two different frequencies, we should find two different values of the angle $\beta$. If these are correctly normalised, taking into account the frequency dependence of $\beta_{0}$, the measurements will yield consistent results for the magnetization. If, on the other hand, the rotations are compared without normalization (or if the frequency dependence of $\beta_{0}$ is neglected), the results will appear inconsistent. In an equilibrium $\mathrm{DyFeO}_{3}$ sample that is $65 \mu \mathrm{m}$ thick, for example, light with a wavelength $\lambda_{0}=0.7 \mu \mathrm{m}$ will be rotated $\beta_{0}=\beta_{\max } \approx 1^{\circ}$, while light with $\lambda_{0}=0.75 \mu \mathrm{m}$ will be rotated only $\beta_{0} \approx 0.5^{\circ}$. If the wrong value of $\beta_{0}$ is used to interpret experimental rotations, estimates of the amplitude of the magnetic oscillations will be wrong by a factor of 2 . If $\beta_{0} \approx 0$ for one of the frequencies used, the error can be arbitrarily large.

This is of particular importance in the case where the magnetic excitation is induced by the same laser source that provides the measurement pulses (as in the inverse Faraday effect experiment described in Ref.[8]). For the same sample and fluence, but with two different laser frequencies, significantly different rotations will be observed. If these are not correctly normalized, it will appear that the induced magnetization is different between the two experiments, i.e. that magnetic excitation via the inverse Faraday effect is strongly dependent on the excitation frequency. This is inconsistent with what is known 
about the inverse Faraday effect (which, being a nonresonant effect, has a very weak dependence on frequency). This example highlights the importance of correct normalization of the measured rotation.

Another problem that may arise due to frequency dependence is that extremely short laser pulses suffer from frequency broadening, and we should repeat the above derivation using a wave packet rather than a plane wave. However, this only becomes necessary when either the frequency broadening of the pulse is very large, or when the frequency dependence of $\delta_{0} d$ is very strong. Fortunately, laser pulses with a time-width of 50 fs do not suffer significant frequency broadening, and are sufficiently short for measuring magnetic oscillations, which have a period of the order of 1 ps. However, if we want to use the Faraday effect to probe faster magnetic dynamics, e.g. dynamics during excitation, we will need shorter pulses, and a wave-packet approach must be used to interpret the results.

\section{NUMERICAL EXPLORATION OF NONPERTURBATIVE REGIONS}

The results of the preceding section may be summarized as follows. For incident light with $\theta \approx 0$, the dependence of the rotation angle on magnetization is linear for small $\kappa$, with the ratio given approximately by the $\kappa=0$ rotation. This "equilibrium rotation" depends both on the material, and on the sample thickness and must be measured for each sample used.

If $\theta$ is not extremely small (i.e. if the condition (20) is not satisfied), the relationship between $\beta$ and $\kappa$ must be determined from Eq.(14), which requires an accurate knowledge of all parameters. Furthermore, the analysis of the previous section does not extend to larger values of $\kappa$. (Recall that since both $m$ and $m_{0}$ are small near magnetic equilibrium, $\kappa$ can be large without violating the assumption that we are near equilibrium.)

To investigate the relationship between rotation and magnetization for large $\theta$ and $\kappa$, we must resort to numerical methods. We calculate $\beta(\kappa)$ from Eq.(14), where $\kappa$ appears (through $\gamma$ ) in both $\delta$ and $\Gamma$. We use the physical constants appropriate for $\mathrm{DyFeO}_{3}$, i.e.

$$
\Gamma_{0} \approx 30, \quad \delta_{0} \approx \frac{\pi}{20} \operatorname{rads} \mu \mathrm{m}^{-1}, \quad \zeta=0.23
$$

To allow easy comparison with experiment, we plot $\Delta \beta \equiv$ $\beta-\beta_{0}$ in degrees. The magnetic deviation $\kappa$ is dimensionless; to emphasize this, we write it as $\kappa=\Delta m / m_{0}$.

Fig. 1 shows the rotation vs. magnetization for $x$ polarized light, propagating through samples of thickness $d=55,60$ and $65 \mu \mathrm{m}$. For $d$ significantly different to $60 \mu \mathrm{m}$, the behaviour is almost linear for all $\kappa$, so that determining magnetization from a measured rotation is easy even for large $\kappa$. The proportionality "constant" between rotation and magnetization changes with thickness, even changing sign (compare the behaviour for
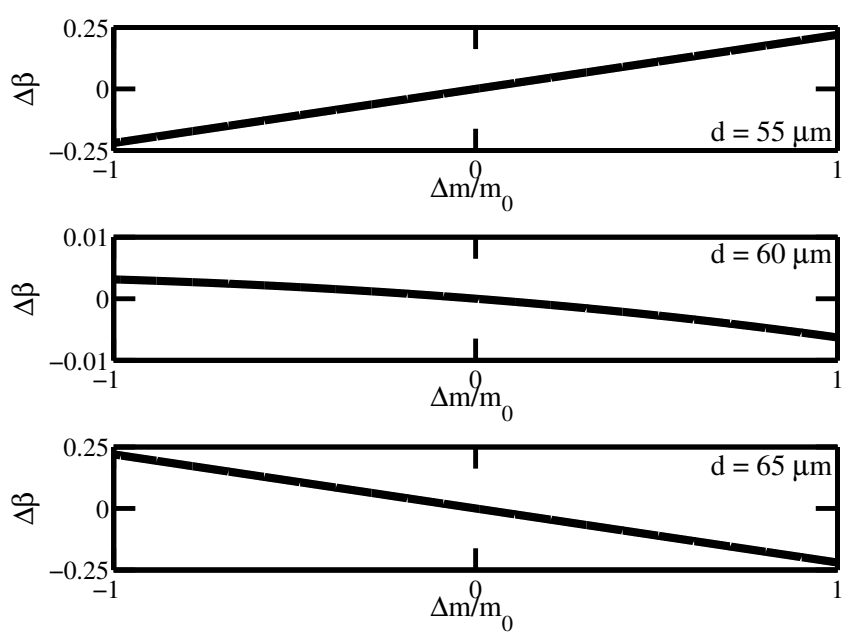

FIG. 1: Comparison of the angle of rotation $\Delta \beta$ (in degrees) with the deviation from magnetic saturation $\left(\kappa=\Delta m / m_{0}\right)$ as a function of sample thickness. Note the change of scale between different plots. In all of these, the incident light is $x$-polarized, i.e. $\theta=0$.
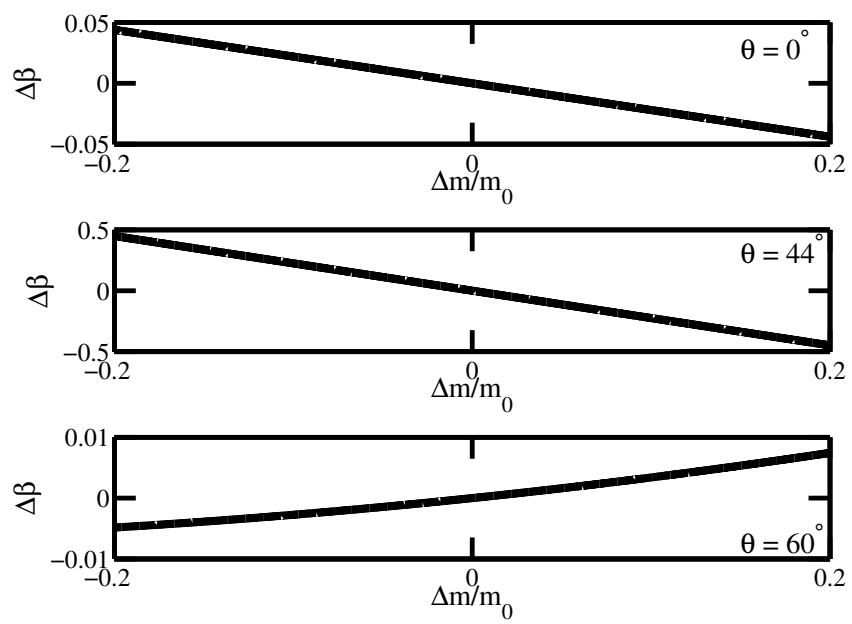

FIG. 2: Comparison of the angle of rotation $\Delta \beta$ (in degrees) with the deviation from magnetic saturation $\left(\kappa=\Delta m / m_{0}\right)$ as the incident polarization angle is changed. The angle $\theta$ is measured in degrees counter-clockwise from the $x$-axis. The sample thickness is kept at $d=65 \mu \mathrm{m}$ throughout. Note that the scale varies between plots.

$d=55 \mu \mathrm{m}$ with $d=65 \mu \mathrm{m})$. In fact, the ratio between changes of $\beta$ and changes of $\kappa$ is very well approximated by $\beta_{0}$, and Eq.(23) is found to be valid even for large values of $\kappa$. This only holds provided we keep $d$ away from the critical regions, and the incident light is assumed to be $x$-polarized. Around $d=60 \mu \mathrm{m}$, the relationship between $\kappa$ and $\beta$ is no longer linear when $\kappa$ not small. In fact the figure is not even symmetric around $\kappa=0$. In this case, symmetric magnetic oscillations will give rise to asymmetric rotation measurements.

Next, we fix $d$ and consider the effect of changing the 
angle $\theta$. We avoid the region where $\beta_{0}$ vanishes by setting $d=65 \mu \mathrm{m}$. Fig. 2 depicts $\Delta \beta$ as a function of $\kappa$ for values of $\theta$ equal to $0^{\circ}, 44^{\circ}$ and $60^{\circ}$. Note that the range of $\kappa$ is much smaller than that of Fig.1, and yet for $\theta=44^{\circ}$ we find Faraday rotation to be as large as $0.5^{\circ}$. We note also that for $\theta=60^{\circ}$, the Faraday rotation is nonlinear and extremely weak; for most other values of $\theta$, the linear approximation is reasonable, although the ratio between $\Delta \beta$ and $\kappa$ is dependent on $\theta$. (In particular, Eq.(23) is a very poor approximation when $\theta \neq 0$.) Measurements with nonzero $\theta$ are useful for experimentally evaluating the values of $\delta_{0}$ and $\Gamma_{0}$.

\section{CONCLUSIONS}

We have investigated Faraday rotation in birefringent magnetically ordered media, and attempted to relate the measurable rotation to the magnetization of the sample. It was found that Faraday rotation is a valid probe for magnetization dynamics, but that care must be taken when interpreting the results. Birefringence breaks the symmetry of the plane transverse to the light propagation direction, so that alignment becomes an important consideration. Furthermore, results must be normalized against the equilibrium rotation, which depends on both the material used and the sample properties (notably, sample thickness).

We have restricted our investigation by ignoring magnetization dynamics in the plane orthogonal to the prop- agation of the probe pulse, ignoring higher order corrections to the dielectric tensor and ignoring diamagnetic terms. The first two of these should have some effect on the rotation, but can only be included at the cost of introducing a number of unknown parameters, which are difficult to estimate. On the other hand, the diamagnetic contribution will have no effect on the rotation unless an external magnetic field is present. If Faraday rotation is used to measure magnetic relaxation after an external field has been switched off, the diamagnetic term may be neglected; however, when trying to determine the magnetooptical parameters of the material using a static magnetic field, the diamagnetic term must be included.

Finally, we have focussed primarily on the rotation and neglected the ellipticity of the outgoing light. Although these contain equivalent information, it is advisable to measure both, and to check that the results are consistent. Inconsistency between these indicate that either the material parameters are not accurate enough, or that an additional effect is present that has been neglected in our analysis.

\section{Acknowledgements}

We would like to thank C. A. Perroni, A. Liebsch and J. Harris for many stimulating discussions. This work was partly funded by the DFG's Schwerpunktprogram 1133 .
[1] C. H. Back et al., Phys. Rev. Lett. 81, 3251 (1998); C. H. Back et al., Science 285, 864 (1999)

[2] M. Bauer et al., Phys. Rev. B 61, 3410 (2000)

[3] H. W. Schumacher et al., Phys. Rev. Lett. 90, 017201 (2003)

[4] S. Kaka and S. E. Russek, Appl. Phys. Lett. 80, 2958 (2002)

[5] E. Beaurepaire, J. C. Merle, A. Daunois and J. Y. Bigot, Phys. Rev. Lett. 76, 4250 (1996)

[6] J. Hohlfeld, E. Matthias, R. Knorren and K. H. Bennemann, Phys. Rev. Lett. 78, 4861 (1997)

[7] A. Scholl, L. Baumgarten, R. Jacquemin and W. Eberhardt, Phys. Rev. Lett. 79, 5146 (1997)

[8] A.V. Kimel, A. Kirilyuk, P. A. Usachev, R. V. Pisarev, A. M. Balbashov and Th. Rasing, Nature 435, 655 (2005)

[9] P. S. Pershan, Phys. Rev. 130, 919 (1963)

[10] J. P. van der Ziel, P.S. Pershan and L. D. Malmstrom, Phys. Rev. Lett. 15, 190 (1965)

[11] C. A. Perroni and A. Liebsch, cond-mat/0602593 (2006)

[12] W.J. Tabor and F.S. Chen, J. Appl. Phys. 40, 2760 (1969).

[13] W.J. Tabor, A.W. Anderson and L.G. van Uitert, J. Appl. Phys. 41, 3018 (1970).

[14] F. J. Kahn, P. S. Pershan and J. P. Remeika, Phys. Rev.
186, 891 (1969)

[15] A. K. Zvezdin and V. A. Kotov, Modern magnetooptics and magnetooptical material, IOP, Bristol (1997)

[16] R. V. Pisarev, Sov. Phys. JETP 31, 761 (1970)

[17] B. B.Krichevtsov, K. M. Mukimov, R. V. Pisarev and M. M. Ruvinshtein, JETP Lett. 34, 379 (1981)

[18] R. M. White, R. J. Nemanich and C. Herring, Phys. Rev. B 25, 1822 (1982)

[19] L. D. Landau and E. M. Lifshitz, Electrodynamics of continuous media, Pergamon, Oxford (1984)

[20] B. B. Krichevtsov, R. V. Pisarev and M. M. Ruvinshtein, Sov. Phys. Solid State 22, 1240 (1981)

[21] A.V. Zenkov, B. B. Krichevtsov, A. S. Moskvin, K. M. Mukimov, R. V. Pisarev and M. M. Ruvinshtein, Sov. Phys. JETP 69, 792 (1989)

[22] N. Koshizuka and K. Hayashi, J. Phys. Soc. Japan 57, 4418 (1988)

[23] P. S. Pershan, J. Appl. Phys. 38, 1482 (1967).

[24] L. D. Landau and E. M. Lifshitz, The classical theory of fields, Butterworth-Heinemann, Oxford (1985)

[25] P. Coeure and D. Challeton, Solid State Commun. 8, 1345 (1970) 